\title{
ON THE $C$-DETERMINANTAL RANGE FOR SPECIAL CLASSES OF MATRICES
}

\author{
ALEXANDER GUTERMAN, RUTE LEMOS, AND GRAÇA SOARES
}

\begin{abstract}
Let $A$ and $C$ be square complex matrices of size $n$, the $C$-determinantal range of $A$ is the subset of the complex plane $\left\{\operatorname{det}\left(A-U C U^{*}\right): U U^{*}=I_{n}\right\}$. If $A, C$ are both Hermitian matrices, then by a result of M. Fiedler [11] this set is a real line segment.

In this paper we study this set for the case when $C$ is a Hermitian matrix. Our purpose is to revisit and improve two well-known results on this topic. The first result is due to C.$\mathrm{K}$. Li concerning the $C$-numerical range of a Hermitian matrix, see Condition 5.1 (a) in [20]. The second one is due to C.-K. Li, Y.-T. Poon and N.-S. Sze about necessary and sufficient conditions for the $C$-determinantal range of $A$ to be a subset of the line, see [21, Theorem 3.3].
\end{abstract}

\section{INTRODUCTION}

Let $M_{n}$ be the algebra of $n \times n$ complex matrices, $U_{n}$ be the group of $n \times n$ unitary matrices and $S_{n}$ be the symmetric group of degree $n$. Let $A, C \in M_{n}$.

Definition 1.1. The $C$-determinantal range of $A$ is the subset of the complex plane denoted and defined by

$$
\triangle_{C}(A)=\left\{\operatorname{det}\left(A-U C U^{*}\right): U \in U_{n}\right\}
$$

and the $C$-determinantal radius of $A$ is

$$
d_{C}(A)=\max \left\{|z|: z \in \triangle_{C}(A)\right\} .
$$

The set $\triangle_{C}(A)$ is compact and connected, but in general it is not convex (see for instance [2, Example 2]) and it may not be simply connected [1]. It is clear that $\triangle_{A}(C)=(-1)^{n} \triangle_{C}(A)$ and this set is unitarily invariant, that is,

$$
\triangle_{C}(A)=\triangle_{V C V^{*}}\left(U A U^{*}\right)
$$

for any $U, V \in U_{n}$.

Definition 1.2. The $\sigma$-points of $\triangle_{C}(A)$ are defined by

$$
z_{\sigma}=\prod_{i=1}^{n}\left(\alpha_{i}-\gamma_{\sigma(i)}\right), \quad \sigma \in S_{n},
$$

where $\alpha_{1}, \ldots, \alpha_{n}$ and $\gamma_{1}, \ldots, \gamma_{n}$ are the eigenvalues of $A$ and $C$, respectively.

It is easy to see that all the (not necessarily distinct) $n ! \sigma$-points belong to $\triangle_{C}(A)$.

The characterization of the $C$-determinantal range of $A$ for Hermitian matrices $A$ and $C$ was obtained by M. Fiedler [11], who proved that $\triangle_{C}(A)$ is a real line segment, whose endpoints are the minimal and maximal $\sigma$-points of $\triangle_{C}(A)$.

The $C$-determinantal range of $A$ is intimately connected with a famous conjecture of M. Marcus [22] and G. N. de Oliveira [24], which can be reformulated as follows: for normal matrices $A, C \in M_{n}$ it holds that $\triangle_{C}(A)$ is a subset of the convex hull of the $\sigma$-points $z_{\sigma}, \sigma \in S_{n}$. This

Key words and phrases. $C$-Determinantal range, $C$-numerical range, Marcus-Oliveira conjecture, $\sigma$-points, real sets

2010 Mathematics Subject Classification. 15A15, 15A60, 15A86 . 
conjecture was proved for $n \leq 3$ and for particular classes of matrices (see $[2,3,5,10]$ and references therein).

The set $\triangle_{C}(A)$ can be considered as a variation of the well-known $C$-numerical range of $A$,

$$
W_{C}(A)=\left\{\operatorname{Tr}\left(A U C U^{*}\right): U \in U_{n}\right\}
$$

which reduces to the classical numerical range $W(A)$ when $C=\operatorname{diag}(1,0, \ldots, 0)$.

The numerical range and its generalizations are related to and have applications in several branches of both pure and applied science. For some incidences of this versatile algebraic tool in quantum theory and related problems, see $[6,12,15,18,25]$.

The main goal of this paper is to investigate the case when the $C$-determinantal and $C$ numerical ranges are real. In particular, we revisit and improve two well-known results on this topic. The first result is due to C.-K. Li and concerns the $C$-numerical range of a Hermitian matrix. In [20, condition 5.1 (a)] it is stated, without a proof, that if $C$ is a real diagonal matrix, then $W_{C}(A) \subset \mathbb{R}$ if and only if $A$ is Hermitian. We show that the above equivalence is not true in general and provide the corresponding counterexamples. Moreover, we find a correct criteria by showing that $W_{C}(A)$ is real if and only if one of the following conditions holds: (i) $A$ is Hermitian or (ii) $\operatorname{Tr} C=0$ and $\operatorname{Im} A$ is a nonzero scalar matrix.

The second result under consideration is due to C.-K. Li, Y.-T. Poon and N.-S. Sze about necessary and sufficient conditions for the $C$-determinantal range of $A$ to be a line segment or a singleton, see [21, Theorems 3.2 and 3.3]. In this paper we characterize matrices $A$ and $C$ for which $C$-determinantal range of $A$ is a subset of the real line. Note that our situation is more specific since in this case $\triangle_{C}(A)$ is automatically either a singleton or a segment, belonging to the real line.

In addition [21, Theorem 3.3] (see also Theorem 4.2 in this paper) contains an equivalence between the fact that the set $\triangle_{C}(A)$ is a line segment which is not a singleton and the set of conditions (see items c1, c2, c3 of Theorem 4.2) concerning the spectrum of $A$ and $C$. Here we obtain a short direct proof of the following implication: the set $\triangle_{C}(A)$ is a line segment which is not a singleton together with the additional assumption that there are no zero $\sigma$-points implies just one of the conditions from the above set, namely condition c1 stating that $A$ and $C$ are both normal matrices.

The paper is organized as follows. In Section 2, we derive some consequences from the elliptical range theorem which characterizes the $C$-determinantal range in the $2 \times 2$ case. In Section 3, the conditions for the $C$-numerical range to be real, when $C$ is assumed to be Hermitian, are investigated. We also study in this section the analogous case for $\triangle_{C}(A)$. In Section 4 , we provide necessary and sufficient conditions for $\triangle_{C}(A)$ to be a nondegenerated real line segment.

\section{Some Consequences of the Elliptical Range Theorem}

The following result called the Elliptical Range Theorem for the $C$-determinantal range provides a complete geometric description of this set for arbitrary 2-square complex matrices [7].

Theorem 2.1. Let $A, C \in M_{2}$ have eigenvalues $\alpha_{1}, \alpha_{2}$ and $\gamma_{1}, \gamma_{2}$, respectively. Then $\triangle_{C}(A)$ is an elliptical disc with foci $\left(\alpha_{1}-\gamma_{1}\right)\left(\alpha_{2}-\gamma_{2}\right)$ and $\left(\alpha_{1}-\gamma_{2}\right)\left(\alpha_{2}-\gamma_{1}\right)$, and minor semi-axis of length $a c-b d$, where $a \geq b$ and $c \geq d$ are the singular values of $A-\frac{1}{2} \operatorname{Tr}(A) I_{2}$ and $C-\frac{1}{2} \operatorname{Tr}(C) I_{2}$, respectively. In particular,

(a) $\triangle_{C}(A)$ is a singleton if and only if $A$ or $C$ is a scalar matrix;

(b) $\triangle_{C}(A)$ is a nondegenerated line segment if and only if $A$ and $C$ are nonscalar normal matrices. 
Next, some easy consequences are derived from the Elliptical Range Theorem.

The Frobenius norm of $A \in M_{n}$ is defined by

$$
\|A\|_{2}=\sqrt{\operatorname{Tr}\left(A^{*} A\right)} .
$$

If $A \in M_{2}$ is a nilpotent matrix then the classical numerical range $W(A)$ is the circular disc centered at the origin with radius $\|A\|_{2}$. If $C \in M_{2}$ has rank one and is not nilpotent, then $W(C)$ is a nondegenerated elliptical disc, its foci are 0 and $\gamma$, its major and minor axis have lengths $\|C\|_{2}$ and $\sqrt{\|C\|_{2}^{2}-|\gamma|^{2}}$, respectively, where $\gamma$ is the nonzero eigenvalue of $C$.

If $A, C \in M_{2}$ and $A$ is nilpotent, then 0 is the only eigenvalue of $A$. It follows readily from Theorem 2.1 that $\triangle_{C}(A)$ is a circular disc centered at the determinant of $C$.

Corollary 2.2. Let $A, C \in M_{2}$ and $A$ be a nonzero nilpotent matrix. If $C$ has rank one, then $\triangle_{C}(A)$ is a nondegenerated circular disc centered at the origin. If additionally

i. $C$ is not nilpotent and $\gamma$ is its nonzero eigenvalue, then the radius of the circular disc is

$$
d_{C}(A)=\|A\|_{2} \frac{\|C\|_{2}+\sqrt{\|C\|_{2}^{2}-|\gamma|^{2}}}{2}
$$

ii. $C$ is nilpotent, then the radius of the circular disc is equal to

$$
d_{C}(A)=\|A\|_{2}\|C\|_{2} .
$$

Proof. If $A$ is nilpotent and $C$ has rank one, hence $C$ has eigenvalue 0 , then it readily follows from Theorem 2.1 that $\triangle_{C}(A)$ is a circular disc centered at the origin and $d_{C}(A)$ is the radius of this disc. By Schur Triangularization Theorem [16, Theorem 2.3.1], the matrices $A$ and $C$ are unitarily similar to

$$
A^{\prime}=\left[\begin{array}{cc}
0 & \alpha \\
0 & 0
\end{array}\right], \quad \alpha \neq 0, \quad \text { and } \quad C^{\prime}=\left[\begin{array}{ll}
0 & \beta \\
0 & \gamma
\end{array}\right], \quad(\beta, \gamma) \neq(0,0),
$$

respectively. Since the spectrum of $X Y$ is equal to the spectrum of $Y X$ for any square matrices $X, Y$, it is easy to see that the singular values of $A$ and $C-\frac{1}{2} \operatorname{Tr}(C) I_{2}$ coincide with those of $A^{\prime}$ and $C^{\prime}-\frac{\gamma}{2} I_{2}$, respectively. The nonzero singular value $a$ of $A^{\prime}$ is equal to $|\alpha|=\|A\|_{2}$. The greatest singular value $c$ of $C^{\prime}-\frac{\gamma}{2} I_{2}$ is given by the square root of

$$
\frac{|\beta|^{2}}{2}+\frac{|\gamma|^{2}}{4}+\frac{1}{2} \sqrt{|\beta|^{4}+|\beta|^{2}|\gamma|^{2}}
$$

Clearly,

$$
\|C\|_{2}^{2}=\operatorname{Tr}\left(C^{*} C^{\prime}\right)=|\beta|^{2}+|\gamma|^{2} \neq 0 \quad \text { and } \quad \sqrt{\|C\|_{2}^{2}-|\gamma|^{2}}=|\beta| \neq 0
$$

Hence, (1) can be written as

$$
\frac{|\beta|^{2}+\|C\|_{2}^{2}+2|\beta|\|C\|_{2}}{4}
$$

and the singular value $c$ of $C$ is the nonzero semi-sum of $\|C\|_{2}$ and $\sqrt{\|C\|_{2}^{2}-|\gamma|^{2}}$.

i. If $C$ is not nilpotent, then $\gamma$ is its nonzero eigenvalue and we get the expression for $d_{C}(A)$.

ii. If $C$ is nilpotent, then $\gamma=0$ and $|\beta|=\|C\|_{2}$. In this case, the radius of the circular disc $d_{C}(A)$ reduces to $\|A\|_{2}\|C\|_{2}$.

A useful technique in the theory of numerical ranges is to reduce problems to the $2 \times 2$ case. For instance, the convexity of $W(A)$ can be proved using such a reduction. The study of the $C$-determinantal range is obviously much more complicated. Nevertheless, the lemma below (see [13, Lemma 2.2] for a proof) provides an efficient reduction tool that together with the Elliptical Range Theorem can lead to some interesting results. 
Let $\operatorname{spec}(X)$ denote the family of all the eigenvalues of $X \in M_{n}$, repeated according to their multiplicities. For $A, C \in M_{n}$ with $\operatorname{spec}(A)=\left\{\alpha_{1}, \ldots, \alpha_{n}\right\}, \operatorname{spec}(C)=\left\{\gamma_{1}, \ldots, \gamma_{n}\right\}$ and $\sigma \in S_{n}$, by Schur Triangularization Theorem, $A$ and $C$ are unitarily similar to upper triangular matrices

$$
A^{\prime}=\left[\begin{array}{cc}
A_{1} & A_{3} \\
0 & A_{2}
\end{array}\right] \quad \text { and } \quad C^{\prime}=\left[\begin{array}{cc}
C_{1} & C_{3} \\
0 & C_{2}
\end{array}\right]
$$

with the main diagonals $\left(\alpha_{1}, \ldots, \alpha_{n}\right)$ and $\left(\gamma_{\sigma(1)}, \ldots, \gamma_{\sigma(n)}\right)$, respectively, where $A_{1}, C_{1} \in M_{2}$ and $A_{2}, C_{2} \in M_{n-2}$ are upper triangular.

Lemma 2.3. [13, Lemma 2.2] Let $A, C \in M_{n}$ and $\sigma \in S_{n}$. If $A^{\prime}, C^{\prime}$ are as above, then

$$
\prod_{i=3}^{n}\left(\alpha_{i}-\gamma_{\sigma(i)}\right) \triangle_{C_{1}}\left(A_{1}\right) \subseteq \triangle_{C}(A),
$$

where the upper triangular submatrices $A_{1}, C_{1} \in M_{2}$ of $A^{\prime}, C^{\prime}$ have eigenvalues $\alpha_{1}, \alpha_{2}$ and $\gamma_{\sigma(1)}, \gamma_{\sigma(2)}$, respectively.

Lemma 2.4. [23, Lemma 1] If $A \in M_{n}$ is a non-normal matrix, then $A$ is unitarily similar to an upper triangular matrix with nonzero entry at the position $(1,2)$.

Theorem 2.5. Let $A \in M_{n}$ be a nilpotent nonzero matrix and $C \in M_{n}$ be a matrix of rank $n-1$. Then 0 is an interior point of $\triangle_{C}(A)$.

Proof. Since rank $(C)=n-1$, we may conclude that 0 is a simple eigenvalue of $C$. Therefore, using Schur Triangularization Theorem and the invariance of $\triangle_{C}(A)$ under unitary similarity transformations of $C$, without loss of generality, we may assume that $C$ is an upper triangular matrix with main diagonal $\left(0, \gamma_{2}, \ldots, \gamma_{n}\right)$ and $\gamma_{i} \neq 0, i=2, \ldots, n$.

Since $A$ is non-normal and nilpotent, by Lemma 2.4 and the unitary invariance of $\triangle_{C}(A)$, we may assume $A$ in upper triangular form with nonzero $(1,2)$ entry and $(0,0, \ldots, 0)$ in the main diagonal. By Lemma 2.3, the inclusion (3) holds where $A_{1}, C_{1} \in M_{2}$ are upper triangular matrices which have eigenvalues 0,0 and $0, \gamma_{2}$, respectively.

Since the $(1,2)$ entry of the nilpotent matrix $A$ is nonzero, then the submatrix $A_{1}$ is a nonzero nilpotent matrix. Since $\gamma_{2} \neq 0$, then $C_{1}$ has rank 1 . Hence, by Corollary 2.2 the set $\triangle_{C_{1}}\left(A_{1}\right)$ is a circular disc centered at the origin and $d_{C_{1}}\left(A_{1}\right) \neq 0$. We may conclude that 0 is an interior point of $\triangle_{C}(A)$, because the product $\prod_{i=3}^{n} \gamma_{i}$ does not vanish.

Denote the boundary of $\triangle_{C}(A)$ by $\partial \triangle_{C}(A)$. In the subsequent discussion, we need the notion of a corner point. We present the formal definition taken from [4].

Definition 2.6. We call $z \in \partial \triangle_{C}(A)$ a corner point of $\triangle_{C}(A)$ if there exists a ball, $B(z, \epsilon)$, with center at $z$ and radius $\epsilon$, such that $B(z, \epsilon) \cap \triangle_{C}(A)$, for $\epsilon>0$ sufficiently small, is contained in a sector limited by two straight lines intersecting at $z$ defining an angle strictly smaller than $\pi$.

The following important fact concerning a corner point is needed below.

Theorem 2.7. [21, Theorem 3.10] Let $A, C \in M_{n}$. Then every corner of $\triangle_{C}(A)$ is a $\sigma$-point of $\triangle_{C}(A)$.

Corollary 2.8. Under the hypothesis of Theorem 2.5, there are no corners on the boundary of $\triangle_{C}(A)$. 
Proof. If there exists a corner $z$ of $\triangle_{C}(A)$, then $z$ is a $\sigma$-point of $\triangle_{C}(A)$. However, all the $\sigma$-points of $\triangle_{C}(A)$ are equal to 0 , because $A$ is nilpotent and $\operatorname{rank}(C)=n-1$. This contradicts the fact proved in Theorem 2.5 that 0 is an interior point of $\triangle_{C}(A)$.

In [21, Theorem 3.3] (see also Theorem 4.2 in this paper), Li, Poon, and Sze contains an equivalence between the fact that the set $\triangle_{C}(A)$ is a line segment which is not a singleton and the set of conditions (see items c1, c2, c3 of Theorem 4.2) concerning the spectrum of $A$ and $C$. Here we obtain a short direct proof of the following implication: the set $\triangle_{C}(A)$ is a line segment which is not a singleton together with the additional assumption that there are no zero $\sigma$-points implies just one of the conditions from the above set, namely condition c1 stating that $A$ and $C$ are both normal matrices. We use the reduction to the $2 \times 2$ case.

Theorem 2.9. Let $A, C \in M_{n}$ be nonscalar matrices. Suppose $z_{\sigma} \neq 0$ for all $\sigma \in S_{n}$. If $\triangle_{C}(A)$ is a line segment, then $A$ and $C$ are both normal matrices.

Proof. If we assume that neither $A$ nor $C$ is normal, then by Lemma 2.4 both matrices $A, C$ are unitarily similar to upper triangular matrices $A^{\prime}, C^{\prime}$ with nonzero entry at the position $(1,2)$. By Lemma 2.3, having in mind that all the $\sigma$-points are assumed to be nonzero, the inclusion

$$
\prod_{i=3}^{n}\left(\alpha_{i}-\gamma_{i}\right) \triangle_{C_{1}}\left(A_{1}\right) \subset \triangle_{C}(A)
$$

holds, where $\alpha_{i}, \gamma_{i}, i=1, \ldots, n$, are the eigenvalues of $A, C$ and $A_{1}, C_{1} \in M_{2}$ are the leading $2 \times 2$ principal submatrices of $A^{\prime}, C^{\prime}$, respectively. Since $A_{1}, C_{1}$ are both non-normal, by the Elliptical Range Theorem, $\triangle_{C_{1}}\left(A_{1}\right)$ is a nondegenerated elliptical disc. The hypothesis that $\triangle_{C}(A)$ is a line segment and the previous inclusion (4) implies that $\triangle_{C_{1}}\left(A_{1}\right)$ is contained in a fixed line, which is a contradiction. Then at least one of the matrices $A, C$ is normal.

Suppose $C$ is normal and $A$ is non-normal, otherwise interchange $A$ and $C$. If $A$ is non-normal, then by Lemma $2.4 A$ is unitarily similar to an upper triangular matrix $A^{\prime}$ with nonzero entry at the position $(1,2)$ and main diagonal $\left(\alpha_{1}, \ldots, \alpha_{n}\right)$. If $C$ is normal, then for any permutation $\sigma \in S_{n}$ the matrix $C$ is unitarily similar to the diagonal matrix $C_{\sigma}$ with the main diagonal $\left(\gamma_{\sigma(1)}, \ldots, \gamma_{\sigma(n)}\right)$. By Lemma 2.3, the inclusion

$$
\Gamma_{\sigma}=\prod_{i=3}^{n}\left(\alpha_{i}-\gamma_{\sigma(i)}\right) \triangle_{C_{1 \sigma}}\left(A_{1}\right) \subset \triangle_{C}(A)
$$

holds where $A_{1}, C_{1 \sigma} \in M_{2}$ are the leading $2 \times 2$ principal submatrices of $A^{\prime}, C_{\sigma}$, respectively. From the hypothesis, we find that $\Gamma_{\sigma}$ is contained in a fixed line and since all the $\sigma$-points are assumed to be nonzero, then $\triangle_{C_{1 \sigma}}\left(A_{1}\right)$ is contained in a fixed line. Since $A_{1}$ is non-normal, it follows by the Elliptical Range Theorem that $\triangle_{C_{1 \sigma}}\left(A_{1}\right)$ is a singleton and hence $C_{1 \sigma}$ is a scalar matrix. Thus $\gamma_{\sigma(1)}=\gamma_{\sigma(2)}$ holds for any $\sigma \in S_{n}$. We may conclude that all the eigenvalues of $C$ are equal. Since by assumption $C$ is normal, it follows that $C$ is a scalar matrix, a contradiction. Hence, both $A$ and $C$ are normal matrices.

\section{On the $C$-Numerical Range and $C$-Determinantal Range when $C$ is HERMITIAN}

For $A, C \in M_{n}$ the $C$-numerical range of $A$ is given by the set

$$
W_{C}(A)=\left\{\operatorname{Tr}\left(A U C U^{*}\right): U \in U_{n}\right\} .
$$

Obviously, $W_{A}(C)=W_{C}(A)$ and the unitary invariance property $W_{C}(A)=W_{V C V^{*}}\left(U A U^{*}\right)$ for any $U, V \in U_{n}$ holds. Westwick [27] proved that for any $A \in M_{n} W_{C}(A)$ is a convex set, when 
$C \in M_{n}$ is a real diagonal matrix. The $\sigma$-points of $W_{C}(A)$ are the elements of this set defined by

$$
w_{\sigma}=\sum_{i=1}^{n} \alpha_{i} \gamma_{\sigma(i)}, \quad \sigma \in S_{n},
$$

where $\alpha_{1}, \ldots, \alpha_{n}$ and $\gamma_{1}, \ldots, \gamma_{n}$ are the eigenvalues of $A$ and $C$, respectively. If $A, C \in M_{n}$ are both normal matrices, then the inclusion of $W_{C}(A)$ in the convex hull of its $\sigma$-points $w_{\sigma}$, $\sigma \in S_{n}$, trivially holds [24]. It follows from Westwick's Theorem that this inclusion becomes equality if one of the normal matrices $A, C$ has collinear eigenvalues. We have that $W_{C}(A)$ is a singleton if and only if $A$ or $C$ is a scalar matrix [19, Theorem 2.5], in which case

$$
W_{C}(A)=\left\{\frac{1}{n} \operatorname{Tr}(A) \operatorname{Tr}(C)\right\} .
$$

In general, the set $W_{C}(A)$ is star-shaped with respect to the point $\frac{1}{n} \operatorname{Tr}(A) \operatorname{Tr}(C)$ for any $A, C \in M_{n}[8]$.

If $C$ is a rank one Hermitian orthogonal projection, then $W_{C}(A)$ reduces to the classical numerical range of $A$,

$$
W(A)=\left\{x^{*} A x: x \in \mathbb{C}^{n}, x^{*} x=1\right\} .
$$

By the celebrated Toeplitz-Hausdorff Theorem $[14,26], W(A)$ is a convex set. It is also called the field of values of $A$ (see [17, Chapter 1] for elementary properties). For instance, $W(A)$ contains the spectrum of $A$ and if $A$ is a normal matrix, then $W(A)$ is the convex hull of the eigenvalues of $A$. In addition, $W(A) \subset \mathbb{R}$ if and only if $A$ is Hermitian.

In [23] M. Marcus and M. Sandy obtained necessary and sufficient conditions for $W_{C}(A)$ to be a subset of the real line.

In [20, condition $5.1(\mathrm{a})]$ it is stated, without a proof, that if $C$ is a real diagonal matrix then $W_{C}(A) \subset \mathbb{R}$ if and only if $A$ is Hermitian. We would like to point out that the above equivalence is not true in general. More precisely, the fact that $W_{C}(A) \subset \mathbb{R}$ may not guarantee that $A$ is Hermitian as the following counterexamples show.

Example 3.1. If $C=I_{2}$ and $A=\operatorname{diag}(i,-i)$, then $W_{C}(A)=\{0\} \subset \mathbb{R}$ and $A$ is not Hermitian.

Example 3.2. Let $C=\operatorname{diag}(1,-1)$ and $A=\operatorname{diag}(1+3 i,-2+3 i)$. Then $W_{C}(A)$ is a line segment joining the $\sigma$-points

$$
w_{1}=1(1+3 i)-1(-2+3 i)=3 \quad \text { and } \quad w_{2}=-1(1+3 i)+1(-2+3 i)=-3 .
$$

Hence $W_{C}(A)=[-3,3] \subset \mathbb{R}$ and $A$ is not Hermitian.

We need the following lemma for further considerations.

Lemma 3.3. [19] Suppose $C$ is a nonscalar normal matrix and $A$ is not normal. Then $W_{C}(A)$ contains a nondegenerated elliptical disc.

The next theorem characterizes the case when $W_{C}(A)$ is a nondegenerated real line segment for an Hermitian matrix $C$. So, it corrects condition 5.1 (a) in [20] and generalizes it for non-diagonal matrices.

Let $\operatorname{Im} A=\frac{A-A^{*}}{2 i}$ denote the imaginary part of $A$.

Theorem 3.4. Let $A, C \in M_{n}$ be nonscalar matrices and $C$ be Hermitian. Then $W_{C}(A) \subset \mathbb{R}$ if and only if one of the following conditions hold:

i. $A$ is Hermitian

ii. $\operatorname{Tr} C=0$ and $\operatorname{Im} A$ is a nonzero scalar matrix. 
Proof. $(\Rightarrow)$ Since $C$ is Hermitian and $W_{C}(A)$ does not contain any nondegenerated elliptical disc, then by Lemma 3.3 the matrix $A$ is normal. Due to the unitary invariance property of the $C$-numerical range, we may suppose $A=\operatorname{diag}\left(\alpha_{1}, \ldots, \alpha_{n}\right)$ and $C=\operatorname{diag}\left(\gamma_{1}, \ldots, \gamma_{n}\right)$ with $\gamma_{1}, \ldots, \gamma_{n} \in \mathbb{R}$ and $\gamma_{1} \neq \gamma_{2}$, because $C$ is not scalar. By definition, all the $\sigma$-points $w_{\sigma}$ belong to $W_{C}(A) \subset \mathbb{R}$. For each pair $(i, j)$ with $i \neq j$, consider $\sigma_{1}, \sigma_{2} \in S_{n}$ such that

$$
\sigma_{1}(i)=1, \sigma_{1}(j)=2, \quad \sigma_{2}(i)=2, \sigma_{2}(j)=1 \quad \text { and } \quad \sigma_{1}(k)=\sigma_{2}(k), \quad k \in\{1, \ldots, n\} \backslash\{i, j\} .
$$

Then

$$
w_{\sigma_{1}}-w_{\sigma_{2}}=\left(\alpha_{i} \gamma_{1}+\alpha_{j} \gamma_{2}\right)-\left(\alpha_{i} \gamma_{2}+\alpha_{j} \gamma_{1}\right)=\left(\alpha_{i}-\alpha_{j}\right)\left(\gamma_{1}-\gamma_{2}\right) \in \mathbb{R}
$$

and $\gamma_{1}-\gamma_{2}$ is a non-zero real number, which implies that $\alpha_{i}-\alpha_{j} \in \mathbb{R}$. If $\alpha_{j} \in \mathbb{R}$, then

$$
\alpha_{i}=\left(\alpha_{i}-\alpha_{j}\right)+\alpha_{j} \in \mathbb{R}, \quad i \neq j .
$$

Therefore, $A$ can not have simultaneously real and non-real eigenvalues. Then either all the eigenvalues of $A$ are real and thus the normal matrix $A$ is Hermitian or all the eigenvalues of $A$ are non-real. If $\alpha_{i} \notin \mathbb{R}, i=1, \ldots, n$, recalling that $\alpha_{i}-\alpha_{j} \in \mathbb{R}$ for each pair $(i, j), i \neq j$, then $\operatorname{Im}\left(\alpha_{i}-\alpha_{j}\right)=0$ and so $\operatorname{Im} \alpha_{i}=\operatorname{Im} \alpha_{j} \neq 0$. We conclude that $\operatorname{Im} A$ is a nonzero scalar matrix, that is,

$$
A=A^{*}+2 i \lambda I_{n}
$$

with $\lambda=\operatorname{Im} \alpha_{i}$. If $z \in W_{C}(A)$, then

$$
z=\operatorname{Tr}\left(C U^{*} A U\right)=\operatorname{Tr}\left(C U^{*}\left(A^{*}+2 i \lambda I_{n}\right) U\right)=\bar{z}+2 i \lambda \operatorname{Tr} C
$$

for some unitary matrix $U$. Since $\lambda \neq 0$, it is clear that $z=\bar{z}$ if and only if $\operatorname{Tr} C=0$.

$(\Leftarrow)$ This condition is easily obtained.

Remark. By excluding the case when $W_{C}(A)$ is a singleton, the previous theorem could also be extracted from [23, Theorem 3] in the case $C$ is Hermitian.

Despite the similarities between $W_{C}(A)$ and $\triangle_{C}(A)$, some crucial differences immediately arise and the difficulty level of $C$-determinantal range problems is always substantially higher.

Similarly to $W_{C}(A)$, when $C$ is Hermitian, the $C$-determinantal range of $A$ can be a subset of the real line even if $A$ is not Hermitian, as the following examples easily show.

Example 3.5. Let $C=I_{2}$ and $A=\operatorname{diag}(i,-i)$. Then $\triangle_{C}(A)=\left\{\operatorname{det}\left(A-I_{2}\right)\right\}=\{2\} \subset \mathbb{R}$ and $A$ is not Hermitian.

Example 3.6. If $C=\operatorname{diag}(2,3)$ and $A=\operatorname{diag}(1-i, 4-i)$, then by Theorem 2.1 the set $\triangle_{C}(A)$ is the real line segment with endpoints -5 and -2 , but $A$ is not Hermitian.

Remark 3.7. In both these examples the eigenvalues of $A$ and $C$ are concyclic, i.e., they belong to the same circle. In Example 3.5 the eigenvalues lie on a circle centered at the origin. In Example 3.6 the eigenvalues lie on a circle centered at $\frac{5}{2}-\frac{3}{2} i$.

Now, let us derive conditions under which, assuming that $C$ is Hermitian and no null $\sigma$-points exist in $\triangle_{C}(A)$, we have that $\triangle_{C}(A)$ is real.

Let us introduce the following Möbius transformation. We denote $\mathbb{C}_{\infty}=\mathbb{C} \cup\{\infty\}$. 
Definition 3.8. For $z_{1}, z_{2}, z_{3}, z_{4} \in \mathbb{C}_{\infty}$ define the Möbius transformation $S: \mathbb{C}_{\infty} \rightarrow \mathbb{C}_{\infty}$ by

$$
\begin{array}{ll}
S(z)=\frac{\left(z-z_{3}\right)\left(z_{2}-z_{4}\right)}{\left(z-z_{4}\right)\left(z_{2}-z_{3}\right)} & \text { if } z_{2}, z_{3}, z_{4} \in \mathbb{C} ; \\
S(z)=\frac{z-z_{3}}{z-z_{4}} & \text { if } z_{2}=\infty ; \\
S(z)=\frac{z_{2}-z_{4}}{z-z_{4}} & \text { if } z_{3}=\infty ; \\
S(z)=\frac{z-z_{3}}{z_{2}-z_{3}} & \text { if } \quad z_{4}=\infty .
\end{array}
$$

Remark 3.9. If $z_{2}, z_{3}, z_{4} \in \mathbb{C}$, then we clearly have

$$
S\left(z_{2}\right)=1, \quad S\left(z_{3}\right)=0 \quad \text { and } \quad S\left(z_{4}\right)=\infty,
$$

and $S$ is the unique Möbius transformation which satisfies (5).

Definition 3.10. The cross ratio of $z_{1}, z_{2}, z_{3}, z_{4} \in \mathbb{C}_{\infty}$ is the image of $z_{1}$ under the unique Möbius transformation $S$, satisfying (5).

Lemma 3.11. [9] Let $z_{1}, z_{2}, z_{3}, z_{4}$ be four distinct points in $\mathbb{C}_{\infty}$. The cross ratio of $z_{1}, z_{2}, z_{3}, z_{4}$ is a real number if and only if $z_{1}, z_{2}, z_{3}, z_{4}$ belong to the same straight line or to the same circle.

Lemma 3.12. [3, Theorem 3] If $A, C \in M_{n}$ are diagonal matrices and their eigenvalues have the same modulus, then $\triangle_{C}(A)$ is a line segment.

Theorem 3.13. Let $A, C \in M_{n}$ be nonscalar matrices. Suppose $z_{\sigma} \neq 0$ for all $\sigma \in S_{n}$ and $C$ is Hermitian. Then $\triangle_{C}(A) \subset \mathbb{R}$ if and only if $f$ the following conditions hold:

i. $A$ is Hermitian

ii. $A$ is normal with the eigenvalues of $A$ and $C$ concyclic, and $z_{\sigma} \in \mathbb{R}$ for all $\sigma \in S_{n}$.

Proof. Let $C \in M_{n}$ be Hermitian.

$(\Leftarrow)$ If $A$ is Hermitian too, then by Fiedler's result $\triangle_{C}(A)$ is a real line segment.

If $A$ is a nonscalar and normal matrix with the eigenvalues $\alpha_{1}, \ldots, \alpha_{n}$ of $A$ and $\gamma_{1}, \ldots, \gamma_{n}$ of $C$ concyclic, that is, $\left|\alpha_{i}-\mu\right|=\left|\gamma_{i}-\mu\right| \neq 0$ for some scalar $\mu$, then the eigenvalues of the unitarily diagonalizable matrices $A_{\mu}=A-\mu I_{n}$ and $C_{\mu}=C-\mu I_{n}$ have the same modulus. By the unitarily invariance property of the determinantal range and by Lemma 3.12 we have that $\triangle_{C}(A)=\triangle_{C_{\mu}}\left(A_{\mu}\right)$ is a line segment. By Lemma 2.7 the endpoints of this segment are $\sigma$-points, which are assumed to be real, then $\triangle_{C}(A) \subset \mathbb{R}$.

$(\Rightarrow)$ Under the hypothesis, all the $\sigma$-points of $\triangle_{C}(A)$ are nonzero and $\triangle_{C}(A)$ is contained in the real line. By Theorem 2.9 the matrix $A$ must be normal.

(i) If the normal matrix $A$ only has real eigenvalues, then $A$ is Hermitian.

(ii) Suppose the opposite, say $\alpha_{1}$ is a non-real eigenvalue of $A$. Let $\gamma_{1}$ be one of the eigenvalues of $C$. If all the eigenvalues of the normal matrix $A$ were equal, then $A$ was scalar, contradicting the hypothesis. Let $\alpha_{2}$ be any eigenvalue of $A$ distinct from $\alpha_{1}$. The Hermitian matrix $C$ is also assumed nonscalar, so let $\gamma_{2}$ be any eigenvalue of $C$ distinct from $\gamma_{1}$. The cross ratio of $\alpha_{1}, \alpha_{2}, \gamma_{1}, \gamma_{2}$ is $S\left(\alpha_{1}\right)$, where $S$ is the unique Möbius transformation such that $S\left(\alpha_{2}\right)=1$, $S\left(\gamma_{1}\right)=0$ and $S\left(\gamma_{2}\right)=\infty$. All the $\sigma$-points belong to $\triangle_{C}(A)$, so that they are all real. Then

$$
S\left(\alpha_{1}\right)=\frac{\left(\alpha_{1}-\gamma_{1}\right)\left(\alpha_{2}-\gamma_{2}\right)}{\left(\alpha_{1}-\gamma_{2}\right)\left(\alpha_{2}-\gamma_{1}\right)}=\frac{z_{\mathrm{id}}}{z_{\tau}} \in \mathbb{R}, \quad \text { where } \tau=(12) .
$$


Since $\alpha_{1}, \alpha_{2}, \gamma_{1}, \gamma_{2}$ are four distinct points in $C_{\infty}$ which are not collinear, by Lemma 3.11 we conclude that they must belong to the same circle. Hence, all the eigenvalues of $A$ and $C$ are concyclic.

\section{When is the $C$-Determinantal Range of $A$ Real?}

In this section we characterize the matrices $A$ and $C$ for which the $C$-determinantal range of $A$ is a subset of the real line. To accomplish this task, we revisit [21, Theorem 3.3].

In [21] C.-K. Li, Y.-T. Poon and N.-S. Sze characterized matrices $A, C \in M_{n}$ for which $\triangle_{C}(A)$ is a singleton.

Theorem 4.1. [21, Theorem 3.2] If $A, C \in M_{n}$ and $n \geq 3$, then the $C$-determinantal range of $A$ is a singleton if and only if one of the following two possibilities hold:

(a) $\operatorname{rank}\left(A-\mu I_{n}\right)+\operatorname{rank}\left(C-\mu I_{n}\right)<n$ for some $\mu \in \mathbb{C}$, in which case $\triangle_{C}(A)=\{0\}$;

(b) $A$ or $C$ is a scalar matrix, in which case $\triangle_{C}(A)=\{\operatorname{det}(A-C)\}$.

In the same paper they characterized matrices $A, C \in M_{n}$ for which $\triangle_{C}(A)$ is a segment which does not reduce to a singleton.

For simplicity, the following notation will be used below: $X_{\mu}=X-\mu I_{n}$ for any $X \in M_{n}$ and $\mu \in \mathbb{C}$.

Theorem 4.2. [21, Theorem 3.3] Let $A, C \in M_{n}$ be such that $\triangle_{C}(A)$ is not a singleton. Then the following conditions are equivalent:

(a) $\triangle_{C}(A)$ has empty interior;

(b) $\triangle_{C}(A)$ is a nondegenerated line segment;

(c) There are at least two distinct $\sigma$-points and one of the following conditions holds:

c1. $A$ and $C$ are normal with eigenvalues lying in the same straight line or circle;

c2. there exists $\mu \in \mathbb{C}$ such that one of the matrices $A_{\mu}$ or $C_{\mu}$ is rank one normal, the other is invertible normal and its inverse has collinear eigenvalues;

c3. there exists $\mu \in \mathbb{C}$ such that $A_{\mu}$ and $C_{\mu}$ are unitarily similar to $\tilde{A} \oplus 0_{n-k}$ and $0_{k} \oplus \tilde{C}$, respectively, with $\tilde{A} \in M_{k}$ and $\tilde{C} \in M_{n-k}$ invertible.

The main result of this section is the following more delicate criteria which provides the characterization of the situation when $\triangle_{C}(A) \subset \mathbb{R}$. Note that this situation is more specific since in this case $\triangle_{C}(A)$ is automatically either a singleton or a segment, belonging to the real line.

Theorem 4.3. Let $A, C \in M_{n}$ be such that $\triangle_{C}(A)$ has at least two distinct $\sigma$-points. Then $\triangle_{C}(A) \subset \mathbb{R}$ if and only if one of the following conditions holds for some complex number $\mu$ :

i. $A, C$ are normal matrices and there exists $k \in\{0,1, \ldots, n-1\}$ such that

$$
\operatorname{spec}(A \oplus C) \subset\left\{\mu+r \mathrm{e}^{i \frac{k \pi}{n}}: r \in \mathbb{R}\right\} ;
$$

ii. A, $C$ are normal matrices with eigenvalues in the same circle centered at $\mu$ and

$$
\sum_{\lambda \in \operatorname{spec}(A \oplus C)} \arg (\lambda-\mu)=\left\{\begin{array}{ll}
0, & n \text { even } \\
\pi, & n \text { odd }
\end{array}(\bmod 2 \pi) ;\right.
$$

iii. $\mu \in \operatorname{spec}(A) \cap \operatorname{spec}(C)$ is an eigenvalue of $A \oplus C$ of multiplicity $n$ and

$$
\sum_{\lambda \in \operatorname{spec}(A \oplus C) \backslash\{\mu\}} \arg (\lambda-\mu)=0 \quad(\bmod \pi) .
$$


Proof. Since $\triangle_{C}(A)$ has at least two distinct $\sigma$-points, then it is not a singleton. If $\triangle_{C}(A) \subset \mathbb{R}$, then $\triangle_{C}(A)$ has empty interior and by Theorem 4.2 it is a nondegenerated line segment, such that one of the conditions $\mathbf{c 1}$, $\mathbf{c} \mathbf{2}$ or $\mathbf{c} 3$ of Theorem 4.2 holds.

Suppose that $\mathbf{c} \mathbf{1}$ holds. If $A, C$ are normal matrices, then without loss of generality we may consider $A=\operatorname{diag}\left(\alpha_{1}, \ldots, \alpha_{n}\right)$ and $C=\operatorname{diag}\left(\gamma_{1}, \ldots, \gamma_{n}\right)$. Since the eigenvalues of $A, C$ are located in the same straight line or the same circle, we divide the proof into two cases.

First Case: Assume that all the eigenvalues lie in the same straight line, that is, there exists $\mu \in \mathbb{C}$ and $\theta \in[0, \pi[$ such that either $\arg (\lambda-\mu)=\theta$ or $\arg (\mu-\lambda)=\theta$ for any $\lambda \in \operatorname{spec}(A \oplus C)$. Then

$$
\begin{aligned}
& \mathrm{e}^{-i \theta} A_{\mu}=\operatorname{diag}\left(\epsilon_{\alpha_{1}}\left|\alpha_{1}-\mu\right|, \ldots, \epsilon_{\alpha_{n}}\left|\alpha_{n}-\mu\right|\right), \\
& \mathrm{e}^{-i \theta} C_{\mu}=\operatorname{diag}\left(\epsilon_{\gamma_{1}}\left|\gamma_{1}-\mu\right|, \ldots, \epsilon_{\gamma_{n}}\left|\gamma_{n}-\mu\right|\right),
\end{aligned}
$$

where $\epsilon_{\lambda}=1$ if $\arg (\lambda-\mu)=\theta$ and $\epsilon_{\lambda}=-1$ if $\arg (\mu-\lambda)=\theta$, are both Hermitian matrices. By Fiedler's result $\triangle_{\mathrm{e}^{-i \theta} C_{\mu}}\left(\mathrm{e}^{-i \theta} A_{\mu}\right)$ is a real line segment, but from the equality

$$
\mathrm{e}^{i n \theta} \triangle_{\mathrm{e}^{-i \theta} C_{\mu}}\left(\mathrm{e}^{-i \theta} A_{\mu}\right)=\triangle_{C_{\mu}}\left(A_{\mu}\right)=\triangle_{C}(A)
$$

and the assumption $\triangle_{C}(A) \subset \mathbb{R}$, we find that $\theta=\frac{k \pi}{n}$ for some $k \in\{0,1, \ldots, n-1\}$, that is, condition i. holds.

Second Case: Assume that all the eigenvalues lie in the same circle centered at $\mu$, that is, $\left|\alpha_{j}-\mu\right|=\left|\gamma_{j}-\mu\right|=\rho, j=1, \ldots, n$. Let $\theta_{j}=\arg \left(\alpha_{j}-\mu\right)$ and $\eta_{j}=\arg \left(\gamma_{j}-\mu\right), j=1, \ldots, n$. Then

$$
A_{\mu}=\rho \operatorname{diag}\left(\mathrm{e}^{i \theta_{1}}, \ldots, \mathrm{e}^{i \theta_{n}}\right), \quad C_{\mu}=\rho \operatorname{diag}\left(\mathrm{e}^{i \eta_{1}}, \ldots, \mathrm{e}^{i \eta_{n}}\right)
$$

and it is easy to see that $A_{\mu} A_{\mu}^{*}=C_{\mu}^{*} C_{\mu}=\rho^{2} I_{n}$. Therefore,

$$
A_{\mu}\left(A_{\mu}^{*}-U C_{\mu}^{*} U^{*}\right)\left(U C_{\mu} U^{*}\right)=A_{\mu} A_{\mu}^{*} U C_{\mu} U^{*}-A_{\mu} U C_{\mu}^{*} C_{\mu} U^{*}=-\rho^{2}\left(A_{\mu}-U C_{\mu} U^{*}\right)
$$

for any $U \in U_{n}$. Consider a nonzero arbitrary point $z \in \triangle_{C}(A)$. Since $\triangle_{C}(A)=\triangle_{C_{\mu}}\left(A_{\mu}\right)$, we have $z=\operatorname{det}\left(A_{\mu}-U C_{\mu} U^{*}\right)$ for some $U \in U_{n}$. From (8) by the properties of the determinant, we get

$$
\operatorname{det}\left(A_{\mu}\right) \bar{z} \operatorname{det}\left(C_{\mu}\right)=(-1)^{n} \rho^{2 n} z \text {. }
$$

By the hypothesis $\triangle_{C}(A) \subset \mathbb{R}$, we have $\bar{z}=z$. The matrices $A, C$ are nonscalar, hence $\rho \neq 0$. By evaluating the determinant of $A_{\mu} C_{\mu}$, we find from (9) that $\mathrm{e}^{i \theta_{1}} \cdots \mathrm{e}^{i \theta_{n}} \mathrm{e}^{i \eta_{1}} \cdots \mathrm{e}^{i \eta_{n}}=(-1)^{n}$. Therefore,

$$
\sum_{j=1}^{n} \theta_{j}+\eta_{j}
$$

is either $0(\bmod 2 \pi)$, when $n$ is even, or $\pi(\bmod 2 \pi)$, when $n$ is odd, and ii. holds.

Assume now that c2 in Theorem 4.2 holds. Suppose that $A_{\mu}$ is a rank one normal matrix and $C_{\mu}$ is an invertible normal matrix whose inverse has collinear eigenvalues for some $\mu \in \mathbb{C}$. Otherwise, interchange $A_{\mu}$ and $C_{\mu}$. The set $\triangle_{C}(A)=\triangle_{C_{\mu}}\left(A_{\mu}\right)$ is not a singleton, then $A_{\mu}, C_{\mu}$ are not scalar. If $A_{\mu}$ has rank $1, C_{\mu}$ is invertible and $A_{\mu}, C_{\mu}$ are both normal matrices, by the unitary invariance of the $C$-determinantal range, we may assume without loss of generality that

$$
A_{\mu}=\operatorname{diag}\left(\alpha_{1}-\mu, 0, \ldots, 0\right), \quad C_{\mu}=\operatorname{diag}\left(\gamma_{1}-\mu, \ldots, \gamma_{n}-\mu\right)
$$

with $\alpha_{1} \neq \mu, \gamma_{j} \neq \mu, j=1, \ldots, n$, and $\gamma_{1}-\mu \neq \gamma_{2}-\mu$. Hence the leading 2-square principal submatrices $A_{\mu}^{\prime}$ of $A_{\mu}$ and $C_{\mu}^{\prime}$ of $C_{\mu}$ are nonscalar diagonal matrices. By the Elliptical Range Theorem, $\triangle_{C_{\mu}^{\prime}}\left(A_{\mu}^{\prime}\right)$ is a nondegenerated line segment with endpoints

$$
\left(\left(\gamma_{1}-\mu\right)-\left(\alpha_{1}-\mu\right)\right)\left(\gamma_{2}-\mu\right) \quad \text { and } \quad\left(\left(\gamma_{2}-\mu\right)-\left(\alpha_{1}-\mu\right)\right)\left(\gamma_{1}-\mu\right) .
$$


By Lemma 2.3, we have

$$
\Upsilon=\prod_{i=3}^{n}\left(\gamma_{i}-\mu\right) \triangle_{C_{\mu}^{\prime}}\left(A_{\mu}^{\prime}\right) \subseteq \triangle_{C_{\mu}}\left(A_{\mu}\right) .
$$

By hypothesis, the eigenvalues $\left(\gamma_{i}-\mu\right)^{-1}$ of $C_{\mu}^{-1}$ are collinear, then there exists $\theta \in[0, \pi[$ such that either $\arg \left(\gamma_{j}-\mu\right)=\theta$ or $\arg \left(\mu-\gamma_{j}\right)=\theta, j=1, \ldots, n$, and $\mathrm{e}^{-i \theta} C_{\mu}$ is Hermitian. Moreover,

$$
\mathrm{e}^{-i n \theta} \Upsilon \subseteq \triangle_{\mathrm{e}^{-i \theta} C_{\mu}}\left(\mathrm{e}^{-i \theta} A_{\mu}\right)=\mathrm{e}^{-i n \theta} \triangle_{C}(A) \subset \mathrm{e}^{-i n \theta} \mathbb{R}
$$

and $\mathrm{e}^{-i n \theta} \Upsilon$ is the line segment with endpoints of the form

$$
\operatorname{det}\left(\mathrm{e}^{-i \theta} C_{\mu}\right)-\left|\alpha_{1}-\mu\right| \frac{\operatorname{det}\left(\mathrm{e}^{-i \theta} C_{\mu}\right)}{\epsilon_{\gamma_{j}}\left|\gamma_{j}-\mu\right|} \mathrm{e}^{i(\kappa-\theta)}, \quad j=1,2,
$$

where $\epsilon_{\gamma_{j}}=1$ if $\arg \left(\gamma_{j}-\mu\right)=\theta, \epsilon_{\gamma_{j}}=-1$ if $\arg \left(\mu-\gamma_{j}\right)=\theta, \kappa=\arg \left(\alpha_{1}-\mu\right)$ and $\operatorname{det}\left(\mathrm{e}^{-i \theta} C_{\mu}\right)$ is a nonzero real number. We find that $\kappa=\theta(\bmod \pi)$, otherwise $\mathrm{e}^{-i n \theta} \Upsilon$ would be a segment of a line not passing through the origin, contradicting the inclusion (10). Hence the matrix e ${ }^{-i \theta} A_{\mu}$ is Hermitian too. As in the proof above of the First Case, it follows from Fiedler's result, the equality (7) and the hypothesis $\triangle_{C}(A) \subset \mathbb{R}$ that i. holds.

At last, assume that $\mathbf{c 3}$ in Theorem 4.2 holds. If there exists $\mu \in \mathbb{C}$, such that $A_{\mu}$ is unitarily similar to $\tilde{A} \oplus O_{n-k}$ and $C_{\mu}$ is unitarily similar to $O_{k} \oplus \tilde{C}$ with $\tilde{A} \in M_{k}, \tilde{C} \in M_{n-k}$ invertible, then $\operatorname{det}(\tilde{A} \tilde{C}) \neq 0$ and $\mu$ is an eigenvalue of $A$ and $C$ of multiplicity $n-k$ and $k$, respectively. By $\left[21\right.$, Lemma 3.6] the set $\triangle_{C_{\mu}}\left(A_{\mu}\right)=\triangle_{C}(A)$ is the line segment joining 0 and $(-1)^{n-k} \operatorname{det}(\tilde{A} \tilde{C})$ and by the hypothesis this nondegenerated line segment is real. Hence, $\operatorname{det}(\tilde{A} \tilde{C})$ given by

$$
\prod_{\lambda \in \operatorname{spec}(A \oplus C) \backslash\{\mu\}}(\lambda-\mu)
$$

must be a nonzero real number and iii. holds.

Conversely, if one of the conditions i., ii. or iii. holds, then by Theorem 4.2 we have that $\triangle_{C}(A)$ is a nondegenerated line segment and it is easy to see that this line segment is real.

Corollary 4.4. Let $A, C \in M_{n}$ be such that $\triangle_{C}(A) \subset \mathbb{R}$. Then there exists a complex scalar $\mu$ such that $A_{\mu} C_{\mu}$ has real determinant.

Proposition 4.5. Let $A, C \in M_{n}$ be nonsingular and $\Lambda$ be the sum of the arguments of all the eigenvalues of $A$ and $C$. Then $\triangle_{C^{-1}}\left(A^{-1}\right) \subset \mathbb{R}$ if and only if $\triangle_{C}(A) \subset e^{i \Lambda} \mathbb{R}$.

Proof. For the sake of simplicity, we denote the product $U C U^{*}$ by $C_{U}$ when $U \in U_{n}$. As a consequence of the identity $A\left(A^{-1}-C_{U}^{-1}\right) C_{U}=-\left(A-C_{U}\right)$, we have

$$
\operatorname{det}(A) \operatorname{det}\left(C_{U}\right) \operatorname{det}\left(A^{-1}-C_{U}^{-1}\right)=(-1)^{n} \operatorname{det}\left(A-C_{U}\right)
$$

for any $U \in U_{n}$. Since $A, C$ are nonsingular, then $\operatorname{det}(A) \operatorname{det}\left(C_{U}\right)=\operatorname{det}(A C)$ is a nonzero number. From the equality

$$
\operatorname{det}(A C) \triangle_{C^{-1}}\left(A^{-1}\right)=(-1)^{n} \triangle_{C}(A),
$$

we easily find that $\triangle_{C^{-1}}\left(A^{-1}\right) \subset \mathbb{R}$ is equivalent to $\triangle_{C}(A) \subset \operatorname{det}(A C) \mathbb{R}$ or to $\triangle_{C}(A) \subset e^{i \Lambda} \mathbb{R}$, because $\operatorname{det}(A C)$ has argument equal to $\Lambda$.

\section{Acknowledgements}

The research contained in this article was started while the first author visited Department of Mathematics, University of Trás-os-Montes and Alto Douro. This author wishes to thank CM-UTAD and the grants RFBR 13-01-00234-a and MD-962.2014.1 for the financial support. 
The work of the second author was supported by portuguese funds through the Center for Research and Development in Mathematics and Applications (CIDMA) and the Portuguese Foundation for Science and Technology (FCT-Fundação para a Ciência e a Tecnologia), within project UID/MAT/04106/2013. The work of the third author was partially supported by FCT (Portugal) with national funds through Centro de Matemática da Universidade de Trás-osMontes e Alto Douro (PEst-OE/MAT/UI4080/2014).

\section{REFERENCES}

[1] N. Bebiano, Some variations on the concept of the c-numerical range, Port. Math. 43 (1985-1986), 189-200.

[2] N. Bebiano, J. K. Merikoski and J. da Providência, On a conjecture of G. N. de Oliveira on determinants, Linear Multilin. Algebra 20 (1987), 167-170.

[3] N. Bebiano and J. da Providência, Some remarks on a conjecture of de Oliveira, Linear Algebra Appl. 102 (1988), 241-246.

[4] N. Bebiano, Yiu-Tung Poon and J. da Providência, On $C$-det spectral and $C$-det convex matrices, Linear Multilin. Algebra 23 (1988), 343-351.

[5] N. Bebiano, A. Kovacec and The validity of the Marcus-de Oliveira Conjecture for essentially Hermitian matrices, Linear Algebra Appl. 197, 198 (1994), 411-427.

[6] N. Bebiano, R. Lemos and J. da Providência, Numerical ranges of unbounded operators arising in quantum physics, Linear Algebra Appl. 381 (2004), 259-279.

[7] N. Bebiano and G. Soares, Three observations on the determinantal range, Linear Algebra Appl. 401 (2005), 211-220.

[8] W. S. Cheung and N. K. Tsing, The $C$-numerical range of matrices is star-shaped, Linear Multilin. Algebra 41 (1996), 245-250.

[9] J. B. Conway, Functions of One Complex Variable I, second ed., Springer Verlag, 1991.

[10] S. W. Drury, Essentially Hermitian matrices revisited, Electronic J. Linear Algebra 15 (2006), 285-296.

[11] M. Fiedler, Bounds for the determinant of the sum of hermitian matrices, Proc. Amer. Math. Soc. 30 (1971), 27-31.

[12] P. Gawron, Z. Puchala, J. A. Miszczak, L. Skowronek and K. Zyczkowski, Restricted numerical range: a versatile tool in the theory of quantum information, J. Math. Physics 51 (2010), 102204 (24 p.).

[13] A. Guterman, R. Lemos and G. Soares, Extremal case in Marcus-Oliveira conjecture and beyond, Linear Multilin. Algebra 61, 3, (2013), 1206-1222.

[14] F. Hausdorff, Der Wertvorrat einer Bilinearform, Math. Z. 3 (1919), 314-316.

[15] U. Helmke, K. Huper and T. Schulte-Herbruggen, Gradient flows computing the $C$-numerical range with applications in NMR Spectroscopy, J. Global Optimization 23 (2002), 283-308.

[16] R. A Horn and C. R. Johnson, Matrix analysis, Cambridge university press, Cambridge, 1985.

[17] R. A Horn and C. R. Johnson, Topics in matrix analysis, Cambridge university press, New York, 1991.

[18] D. W. Kribs, A. Pasieka, M. Laforest, C. Ryan and M. P. Silva, Research problem on numerical ranges in quantum computing, Linear Multilin. Algebra 57 (2009), 491-502.

[19] C.-K. Li, The $C$-convex matrices, Linear Multilin. Algebra 21 (1987), 303-312.

[20] C.-K. Li, $C$-numerical ranges and $C$-numerical radii, Linear Multilin. Algebra 37 (1994), 51-82

[21] C.-K. Li, Y.-T. Poon and N.-S. Sze, Ranks and determinants of the sum of matrices from unitary orbits, Linear Multilin. Algebra 56 (2008), 105-130.

[22] M. Marcus, Derivations, Plücker relations and the numerical range, Indiana Univ. Math. J. 22 (1973), $1137-1149$.

[23] M. Marcus and M. Sandy, Conditions for the generalized numerical range to be real, Linear Algebra Appl. 71 (1985), 219-239.

[24] G. N. de Oliveira, Normal matrices (research problem), Linear Multilin. Algebra 12 (1982), 153-154.

[25] T. Schulte-Herbruggen, G. Dirr, U. Helmke and S. Glaser, The significance of the $C$-numerical range and the local $C$-numerical range in quantum contol and quantum information, Linear Multilin. Algebra 56 (2008), 3-26.

[26] O. Toeplitz, Das algebraische Analogon zu einem Satz von Fejer, Math. Z. 2 (1918), 187-197.

[27] R. Westwick, A theorem on numerical range, Linear Multilin. Algebra 2 (1975), 311-315. 
(Alexander Guterman) Faculty of Algebra, Department of Mathematics and Mechanics, Moscow State University, GSP-1, 119991 Moscow, Russia.

E-mail address, Alexander Guterman: guterman@list.ru

(Rute Lemos) Cidma, Mathematics Department, University of Aveiro, 3810-193 Aveiro, PorTUGAL

E-mail address, Rute Lemos: rute@ua.pt

(Graça Soares) Universidade de Trás-os-Montes e Alto Douro, Utad, Escola das Ciências e Tecnologia, Quinta dos Prados, 5000-801 Vila Real, Portugal, www.utad.Pt

E-mail address, Graça Soares: gsoares@utad.pt 